\title{
An analysis of indirect genetic effects on adult body weight of the Pacific white shrimp Litopenaeus vannamei at low rearing density
}

Sheng Luan ${ }^{1,2}$, Kun Luo ${ }^{1}$, Zhan Chai ${ }^{1}$, Baoxiang Cao ${ }^{1}$, Xianhong Meng ${ }^{1}$, Xia Lu' ${ }^{1}$ Ning Liu' ${ }^{1}$, Shengyu Xu and Jie Kong ${ }^{1,2^{*}}$

\begin{abstract}
Background: Our aim was to estimate the genetic parameters for the direct genetic effect (DGE) and indirect genetic effects (IGE) on adult body weight in the Pacific white shrimp. IGE is the heritable effect of an individual on the trait values of its group mates.

Methods: To examine IGE on body weight, 4725 shrimp from 105 tagged families were tested in multiple small test groups (MSTG). Each family was separated into three groups (15 shrimp per group) that were randomly assigned to 105 concrete tanks with shrimp from two other families. To estimate breeding values, one large test group (OLTG) in a $300 \mathrm{~m}^{2}$ circular concrete tank was used for the communal rearing of 8398 individuals from 105 families. Body weight was measured after a growth-test period of more than 200 days. Variance components for body weight in the MSTG programs were estimated using an animal model excluding or including IGE whereas variance components in the OLTG programs were estimated using a conventional animal model that included only DGE. The correlation of DGE between MSTG and OLTG programs was estimated by a two-trait animal model that included or excluded IGE.

Results: Heritability estimates for body weight from the conventional animal model in MSTG and OLTG programs were $0.26 \pm 0.13$ and $0.40 \pm 0.06$, respectively. The log likelihood ratio test revealed significant IGE on body weight. Total heritable variance was the sum of direct genetic variance (43.5\%), direct-indirect genetic covariance (2.1\%), and indirect genetic variance (54.4\%). It represented $73 \%$ of the phenotypic variance and was more than two-fold greater than that (32\%) obtained by using a classical heritability model for body weight. Correlations of DGE on body weight between MSTG and OLTG programs were intermediate regardless of whether IGE were included or not in the model.

Conclusions: Our results suggest that social interactions contributed to a large part of the heritable variation in body weight. Small and non-significant direct-indirect genetic correlations implied that neutral or slightly cooperative heritable interactions, rather than competition, were dominant in this population but this may be due to the low rearing density.
\end{abstract}

\footnotetext{
*Correspondence: kongjie@ysfri.ac.cn

${ }^{1}$ Key Laboratory for Sustainable Utilization of Marine Fisheries Resources, Ministry of Agriculture, Yellow Sea Fisheries Research Institute, Chinese Academy of Fishery Sciences, Nanjing Road 106, Qingdao 266071, China Full list of author information is available at the end of the article
} 


\section{Background}

Social interactions between individuals have been extensively studied in animal and plant populations [1]. Such interactions may be due to a number of factors, including competition for limited resources (e.g., soil nutrients or food), social behaviors (e.g., aggression, social dominance, competitive ability, or helping behavior), or interactions between mothers and their offspring (maternal effects) [2]. Cannibalistic and aggressive behaviors were often reported in shrimp [3, 4], especially when the stocking density was high and feeding frequency was low. Such social interactions may affect the growth, survival and uniformity of the shrimp. It is difficult to improve the socially affected traits by classical selection methodologies that target only the direct genetic effect (DGE) of an individual on its own phenotype. Unfortunately, it is unrealistic to observe and record social behaviors among shrimp directly because of their small size and high stocking density as well as the complex water environment and time-consuming nature of the procedure. Therefore, in general, social interactions are ignored in most selective breeding programs. Ignoring the indirect genetic effect (IGE) of an individual on the phenotype of its group mates may result in a reversal of the direction of the selection response. In a population of Japanese quails, negative selection responses for 43-day body weight and mortality were obtained when selection of candidates was based on traditional estimated breeding values (EBV) [1]. Such negative selection responses occur because, in classical selection programs, the best individuals may have negative genetic effects on other individuals.

Extended quantitative genetic models have been developed to estimate DGE and IGE without the need for behavioral observation. Individual IGE can be predicted as a random effect in the mixed model using a test design that is capable of detecting a social effect. In the Atlantic cod Gadus morhua, the genetic parameters of DGE and IGE on growth and fin damage traits were estimated using the 3FAM design that consists of 100 small groups of three families [5]. IGE on harvest weight were estimated using an optimal design of multiple blocks of 11 families in the Nile tilapia Oreochromis niloticus [6, 7]. The total genetic variation that underlies a sociallyaffected trait can also be captured using such methods. For example, in the Atlantic cod, the heritable variance for length of the first dorsal fin that included variance of IGE was equal to $28.43 \pm 6.60$ and was more than threefold greater than the additive genetic variance estimated by traditional methods $(8.50 \pm 0.147)$ [5]. Moreover, response to selection for socially affected traits can be increased using group selection or multilevel selection that takes IGE into account [8-13].
The Pacific white shrimp, Litopenaeus vannamei, is an important farmed penaeid shrimp that accounts for $42 \%$ of the total shrimp production in the world [14]. Several classical selective breeding programs have been carried out and resulted in genetic gain for growth and disease resistance [15-19]. However, little is known regarding IGE in farmed shrimp. In this study, our aim was to estimate the genetic parameters of the DGE and IGE on adult body weight at a low rearing density in the Pacific white shrimp. Data from multiple small test groups (MSTG) was analyzed using both a classical animal model and an extended animal model that included DGE and IGE. Moreover, total heritable variance was estimated for each model and then compared. The correlation between the DGE obtained with MSTG and traditional EBV from one large test group (OLTG) was calculated to evaluate the accuracy of the predicted breeding value from MSTG.

\section{Methods}

\section{Ethical statement}

This research was approved by the Animal Care and Use committee in the Yellow Sea Fisheries Research Institute, Chinese Academy of Fishery Sciences; all shrimp were reared and tested at a low rearing density. All in vivo experiments in this study were performed in accordance with the Animal Research: Reporting in Vivo Experiments (ARRIVE) guidelines. Animals used in this study belonged to the Pacific white shrimp L. vannamei species, which originated from Latin American countries, including Brazil, Mexico, and Colombia. This shrimp was introduced into China for farming approximately in 1998 and is neither an endangered nor a protected species both in China and its native countries. All the experiments carried out in this study were in accordance with the Law of the People's Republic of China on the Protection of Wildlife (http://www.china.org.cn/english/environment/34349.htm).

\section{Origin of the base population and selection procedure}

The breeding program was performed at Hainan Higene Aquaculture Technology Ltd (longitude 110.952874, latitude 19.937534) in Wenchang City, Hainan Province, China, in 2012. The founder broodstocks were from eight improved batches that were introduced from different companies in the United States and Singapore. Individuals were checked for different virus strains using reverse transcriptase polymerase chain reaction and only the virus-free individuals were used. After isolation, each shrimp was individually tagged using numbered rings that were placed on one ocular peduncle. Broodstocks with a healthy appearance and mature gonads were chosen after one month of temporary rearing. The base population $\left(\mathrm{G}_{0}\right.$ generation) was formed by an incomplete 
diallel cross experiment for eight strains. A total of 207 full-sib families (40 paternal half-sib families; 59 maternal half-sib families) from 187 sires and 174 dams were obtained by artificial insemination in 2012. These families were tagged using visible implant fluorescent elastomer (VIE) and communally reared in two $100 \mathrm{~m}^{2}$ tanks for the grow-out test.

Breeding candidates in generation $\mathrm{G}_{0}$ were ranked using a selection index [20], which included individual EBV for body weight, and the family EBV for survival. The mating schemes for the selection and control populations in generation $G_{1}$ were created as follows. The selection population was created by mating primarily selected males and females with a high selection index $(>100)$ from families with a high selection index $(>100)$, with restrictions to control inbreeding (e.g., avoiding fullsib, half-sib and cousin mating), and maintaining a large effective population size [21]. The control population was composed of 10 to 20 full-sib families, which were produced from 20 to 30 breeding candidates of generation $G_{0}$ by single-pair mating. A total of 105 families ( 10 paternal half-sib families; 26 maternal half-sib families) from 100 males and 91 females of generation $G_{0}$ were selected to produce generation $\mathrm{G}_{1}$ (Table 1 ).

\section{Programs to estimate the DGE and IGE MSTG program}

The 3FAM design was used to estimate the DGE and IGE on body weight in generation $G_{1}[5,22]$. At the beginning of the experiment, each of the 105 families with 45 shrimp (4725 individuals) was tagged using VIE and equally divided into three groups when the average body weight was about $7 \mathrm{~g}$. Next, each of the three groups from each family were randomly assigned to one of 105 concrete tanks $(170 \mathrm{~cm} \times 170 \mathrm{~cm} \times 100 \mathrm{~cm})$ of approximately $3 \mathrm{~m}^{3}$, with restrictions to control the coancestry coefficient (e.g., avoiding assigning half-sib and cousin families in the same tank) and decrease the coefficient of variation of body weight at tagging between the three families in a tank. In this design, each family was tested against six other families in three different tanks. Each tank contained 45 shrimp from three families that resulted in an average shrimp density of about 15 individuals $/ \mathrm{m}^{2}$ at the beginning of the experiment. All shrimp were stocked in 105 tanks during five days, and were harvested over two days after a growth-test period of 217 days. The water exchange rate for each tank was about 20 to $30 \%$ over 3 days. At the end of the experiment, the average density was about 14 individuals $/ \mathrm{m}^{3}$.

\section{OLTG program}

To obtain the classical EBV and evaluate the genotype by environment interaction effects between the MSTG and
OLTG programs, a circular concrete tank $\left(300 \mathrm{~m}^{2}\right)$ was used for communal rearing of 105 families as used in MSTG at generation $G_{1}$. The progeny of each family were tagged using VIE and mixed for the grow-out test when the average body weight reached about $7 \mathrm{~g}$. The stocking family size ranged from 14 to 90 individuals and was on average equal to 80 . The 8398 individuals were stocked during eight days, and harvested over 6 days after a growth-test period of 216 days. The water exchange rate was only about 5 to $10 \%$ over 7 days because this tank was equipped with a water recirculating system. At the end of the experiment, the average density was about 22 individuals $/ \mathrm{m}^{2}$.

\section{Statistical analysis}

Genetic analysis of body weight was performed using ASReml4 [23]. The data were first analyzed using a traditional animal model without IGE.

The following models were used.

For the MSTG program:

$$
\begin{aligned}
y_{i k l m}= & \mu+\operatorname{Sex}_{l}+\operatorname{Age}_{m}\left(\operatorname{Sex}_{l}\right) \\
& +a_{d_{i}}+c_{m}+t_{k}+e_{i k l m} ;
\end{aligned}
$$

For the OLTG program:

$$
y_{i l m}=\mu+\operatorname{Sex}_{l}+T B W_{m}\left(\operatorname{Sex}_{l}\right)+a_{d_{i}}+e_{i l m} ;
$$

For the MSTG program when both the DGE and IGE were included:

$$
\begin{aligned}
y_{i j k l m}= & \mu+\operatorname{Sex}_{l}+\operatorname{Age}_{m}\left(\operatorname{Sex}_{l}\right)+a_{d_{i}} \\
& +\sum_{i \neq j}^{n-1} a_{s_{j}}+c_{m}+t_{k}+e_{i j k l m} ;
\end{aligned}
$$

where $y_{i j k l m}, y_{i k l m}$ or $y_{i l m}$ is the observed body weight of the $i$ th shrimp; $\mu$ is the overall mean; $S_{e x}$ is the fixed effect of the $l$ th gender; $\operatorname{Age}_{m}\left(\operatorname{Sex}_{l}\right)$ is a linear covariate of age nested within the $l$ th gender; $T B W_{m}\left(\operatorname{Sex}_{l}\right)$ is a covariate of body weight at tagging, with the 'spline' function that uses the unique data values as the knot points in ASReml; $a_{d_{i}}$ is the DGE of the $i$ th shrimp, $a_{d} \sim\left(0, \mathbf{A} \sigma_{a_{d}}^{2}\right)$, where $\mathbf{A}$ is the additive genetic relationship matrix among all shrimp included in generation $G_{1}$ and its parents, and $\sigma_{a_{d}}^{2}$ is the variance of DGE; $\sum_{i \neq j}^{n-1} a_{s_{j}}$ is the sum of the IGE of $\mathrm{n}-1$ associates in the same group as the $i$ th focal individual $(\mathrm{n}=45), a_{\mathrm{s}} \sim\left(0, \mathbf{A} \sigma_{a_{s}}^{2}\right)$, where $\sigma_{a_{\mathrm{s}}}^{2}$ is the variance of IGE; $c_{m}$ is the random effect common to the $m$ th full-sib family, $\mathrm{c} \sim\left(0, \mathbf{I} \sigma_{c}^{2}\right)$, which is a combination of the tank effect due to separate rearing of the full-sib families before stocking and one quarter of the non-additive (dominance) genetic effect common to fullsibs, where $\mathbf{I}$ is the identity matrix, and $\sigma_{c}^{2}$ is the variance of common environmental effect; $t_{k}$ is the random effect of the $k$ th test tank, $t \sim\left(0, \mathbf{I} \sigma_{t}^{2}\right), \sigma_{t}^{2}$ is the variance of 


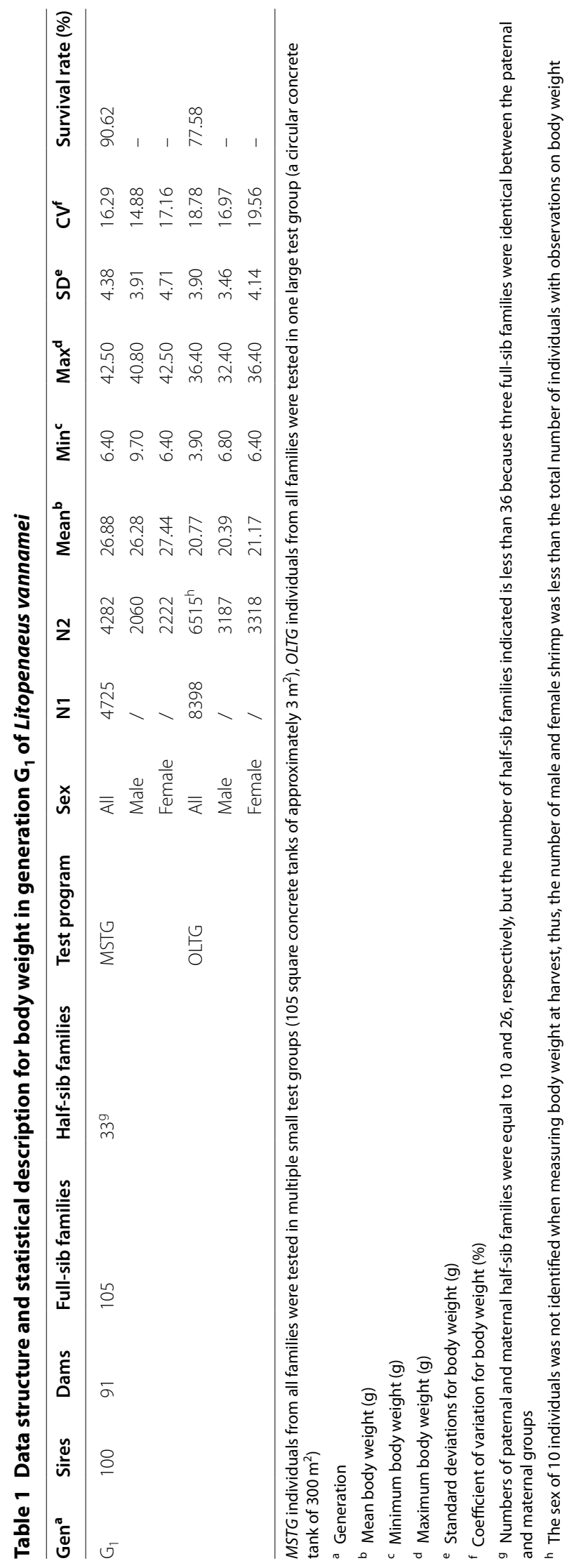


the test tank effect; and $e_{i j k l m}, e_{i k l m}$ or $e_{i l m}$ is the random residual error of the $i$ th individual, $e \sim\left(0, \mathbf{I} \sigma_{e}^{2}\right)$, where $\sigma_{e}^{2}$ is the residual variance.

The $\log$ likelihood ratio test showed that the $c$ and $t$ effects should have been included in Models 1 and 3 . However, it did not converge when the $c$ effect was included in Model 2. Therefore, body weight at tagging rather than age was fitted as the covariate to reduce the impact of common environmental effect in Model 2.

The total breeding value (TBV) was defined as the total heritable effect of an individual on trait values in the population, and was the breeding value of interest for response to selection [9].

$$
T B V_{i}=a_{d_{i}}+(n-1) a_{s_{i}} .
$$

Therefore, the variance of TBV $\left(\sigma_{T B V}^{2}\right)$ was calculated as:

$$
\sigma_{T B V}^{2}=\sigma_{a_{d}}^{2}+2(n-1) \sigma_{a_{d s}}+(n-1)^{2} \sigma_{a_{s}}^{2},
$$

where $\sigma_{a_{d s}}$ is the covariance between the DGE and IGE.

The phenotypic variance $\left(\sigma_{p}^{2}\right)$ was calculated as:

$$
\sigma_{p}^{2}=\sigma_{a_{d}}^{2}+(n-1) \sigma_{a_{s}}^{2}+\sigma_{c}^{2}+\sigma_{t}^{2}+\sigma_{e}^{2} .
$$

In classical quantitative genetic theory, the heritability measure of the direct genetic variance relative to the phenotypic variance is calculated by $h^{2}=\sigma_{a_{d}}^{2} / \sigma_{p}^{2}$. By analogy, to express the heritable total variance to the phenotype variance, $T^{2}$ is introduced as the ratio of $\sigma_{T B V}^{2}$ to $\sigma_{p}^{2}$. In this study, $T^{2}$ only represents the heritable variance expressed on the scale of phenotypic variance among tested shrimp. Comparison of $T^{2}$ and $h^{2}$ gives a quick indication of the contribution of social effects to heritable variance.

The correlation of the DGE on body weight between the MSTG and OLTG programs was estimated by a bivariate animal model including or excluding IGE, where body weight in each program was treated as a separate trait. The $c$ effect was not included in the models, and the residual covariance between the MSTG and OLTG programs was set to zero because some individuals differed between the two programs.

To account for heterogeneity due to the eight strains introduced, which may influence the genetic parameters for body weight and survival [24], eight genetic groups were included in the pedigree and considered using the !GROUP qualifier in ASReml.

\section{Results}

\section{Descriptive statistics}

The coefficients of variation for body weight for the MSTG and OLTG programs were equal to 16.29 and
$18.78 \%$, respectively. The small tank size ( 3 vs. $300 \mathrm{~m}^{2}$ ) may have increased uniformity among individuals that could share the same environment and food. The mean and standard error of body weight for females were slightly greater than those observed for males. The average body weight $(20.77 \mathrm{~g})$ and survival rate $(77.58 \%)$ for the OLTG program were lower than those (26.88 $\mathrm{g}$ and $90.62 \%)$ for the MSTG program. The water recirculating system that equipped the tank used in the OLTG program may have been responsible for this trend.

\section{Variance components and genetic parameters}

Table 2 shows variance components and genetic parameters for body weight that were estimated using the conventional animal model (Models 1 and 2). Heritabilities of $0.26 \pm 0.13$ and $0.40 \pm 0.06$ for body weight were obtained for the MSTG and OLTG programs, respectively. The latter was likely biased upward because the $c$ effect was not fitted in Model 2. For the MSTG program, the $\mathrm{c}^{2}$ for body weight was small $(0.07 \pm 0.06)$ and not significant $(P<0.05)$. The variance of the test tank explained $20.90 \%$ of the phenotypic variance. The estimated correlation of the DGE of body weight between the MSTG and OLTG programs was equal to $0.71 \pm 0.11$.

Table 2 Genetic parameters estimated using two animal models for body weight in Litopenaeus vannamei

\begin{tabular}{llllc}
\hline Parameters & \multicolumn{2}{l}{ Animal model without IGE } & & \multicolumn{2}{l}{ Animal model with IGE } \\
\cline { 2 - 3 } & MSTG & OLTG & & MSTG \\
\hline$\sigma_{a_{d}}^{2}$ & $4.74 \pm 2.55$ & $6.26 \pm 1.05$ & & $5.06 \pm 2.46$ \\
$\sigma_{a_{s}}^{2}$ & - & - & - & $0.00395 \pm 0.00282$ \\
$\sigma_{a_{d s}}$ & - & - & $0.00309 \pm 0.0386$ \\
$\sigma_{c}^{2}$ & $1.29 \pm 1.03$ & - & $1.01 \pm 0.96$ \\
$\sigma_{t}^{2}$ & $3.81 \pm 0.61$ & - & $1.46 \pm 0.57$ \\
$\sigma_{T B V}^{2}$ & - & - & $11.63 \pm 5.81$ \\
$\sigma_{e}^{2}$ & $8.34 \pm 1.30$ & $9.22 \pm 0.58$ & $8.18 \pm 1.26$ \\
$\sigma_{p}^{2}$ & $18.19 \pm 0.92$ & $15.48 \pm 0.57$ & & $15.88 \pm 0.84$ \\
$T^{2}$ & - & - & $0.73 \pm 0.37$ \\
$h^{2}$ & $0.26 \pm 0.13$ & $0.40 \pm 0.06$ & & $0.32 \pm 0.15$ \\
$c^{2}$ & $0.07 \pm 0.06$ & - & $0.06 \pm 0.06$ \\
$r_{a_{d s}}$ & - & - & $0.02 \pm 0.26$ \\
$r_{g}$ & $0.71 \pm 0.11$ & & $0.68 \pm 0.11$ \\
\hline
\end{tabular}

IGE indirect genetic effects, MSTG individuals from all families were tested in multiple small test groups ( 105 square concrete tanks of approximately $3 \mathrm{~m}^{2}$ ), OLTG individuals from all families were tested in one large test group (a circular concrete tank of $\left.300 \mathrm{~m}^{2}\right), \sigma_{a_{d}}^{2}$ direct genetic variance, $\sigma_{a_{\mathrm{s}}}^{2}$ indirect genetic variance, $\sigma_{a_{d s}}$ direct-indirect genetic covariance, $\sigma_{c}^{2}$ common environmental variance, $\sigma_{t}^{2}$ variance of the test tank effect, $\sigma_{T B V}^{2}$ variance of the total breeding value, $\sigma_{e}^{2}$ residual variance, $\sigma_{p}^{2}$ phenotypic variance, $T^{2}$ ratio of $\sigma_{T B V}^{2}$ to $\sigma_{p}^{2}, h^{2}$ heritability, $c^{2}$ common environmental coefficient, $r_{a d s}$ correlation between the direct genetic effect (DGE) and IGE, $r_{g}$ genetic correlation on DGE between the MSTG and OLTG programs 
For the MSTG program, IGE were found to be significant with the log likelihood ratio test (LRT) between Models 1 and 3 being equal to $86.58(P<0.001)$. Direct genetic variance was not significantly affected by the inclusion of IGE in Model 3 (Table 2). The small indirect genetic variance $(0.00395 \pm 0.00282)$ contributed substantially to the heritable variance. The estimated ratio $\left(\mathrm{T}^{2}\right)$ of the total heritable variance over the phenotypic variance was $0.73 \pm 0.37$, which was more than two-fold that of classical heritability $(0.32 \pm 0.15)$. The estimated correlation between the DGE and IGE was small and not significant. The inclusion of IGE reduced estimated tank effects (Table 2) which indicated that tank effects partly originated from social interactions among individuals, rather than entirely from physical differences between tanks. Including IGE in the model only slightly reduced the estimated correlation of the DGE on body weight between the MSTG and OLTG programs $(0.68 \pm 0.11)$ (Table 2) because the values of the IGE were small compared to those of DGE. In this study, standard errors of the variance components and genetic parameters related to IGE were in general large because the amount of data was relatively limited.

\section{Discussion}

In this study, the DGE and IGE on body weight at harvest were estimated for a shrimp L. vannamei population. The heritable variance including that of IGE represented $73 \%$ of the phenotypic variance and was more than twofold greater than that expected with a classical heritability analysis, which indicates that social interactions may contribute to a large part of the heritable variation. This result suggests that growth rate is strongly influenced by social interactions.

Genetic parameters for socially-affected traits have been estimated in several previous studies on natural or selected populations [25]. Social interactions have a substantial effect on the total heritable variance and explain 6 to $98 \%$ of this variance [25]. For example, for Nile tilapia, it was reported that indirect genetic variance of body weight represented $48 \%$ of the total heritable variance [6]. For the length of the first dorsal fin in farmed Atlantic cod, social interactions explained $45.4 \%$ of the total heritable variation; direct genetic variance only accounted for $21.5 \%$ of the total heritable variation [5]. However, no significant IGE were detected for growth traits in the same 6-week experiment.

In our study, we found small and non-significant direct-indirect genetic correlations, which suggested that heritable interactions in this population were not competitive, but were neutral or slightly cooperative. However, expression of social interactions may depend on the environmental conditions. The low rearing density and the strategy used to reduce the initial variation in body weight at tagging between families in the same tank may have reduced competition between individuals. High rearing density and restricted feeding may create strong competition among mates and result in a negative direct-indirect genetic correlation. Muir [1] reported a negative correlation $(-0.56)$ in a Japanese quail population when feeding was restricted. A negative direct-indirect genetic correlation $(-0.38 \pm 0.19)$ was also found for body weight in the Nile tilapia, which resulted in the total heritable variance being smaller than the additive genetic variance [6].

For the MSTG program, the heritability estimated for body weight using the classical animal model was in line with the estimates reported for other shrimp selective breeding programs $[18,26,27]$. For the OLTG program, the heritability estimated for body weight was greater than those reported in the literature and was likely overestimated because the $c$ effect was not included in Model 2 due to the problem of convergence. One explanation is that the OLTG dataset may not have possessed sufficient depth in the relationships between individuals for the mixed model to separate the common environmental effect because of weak genetic ties between the families (half-sib families accounted for only $31 \%$ of families). However, the $c$ effect was successfully estimated using the same pedigree in the MSTG dataset. In the MSTG program, individuals of each family were assigned to three different tanks and were communally reared with individuals of the other six families. Thus, compared to the OLTG program, in this case, the $c$ effect from each full-sib family was not entirely confounded with the genetic effect and the tank effect due to this special data structure.

The estimated correlation of the DGE on body weight between the MSTG and OLTG programs was around 0.7 and this estimate was hardly affected by including IGE in the model. Other studies have reported higher $(>0.80)$ genetic correlations of body weight between tank environments for shrimp populations [18, 27]. However, in our study, there were probably large environmental differences between the MSTG and OLTG programs. First, the tank size $\left(3 \mathrm{~m}^{2}\right)$ in the MSTG program was much smaller than that $\left(300 \mathrm{~m}^{2}\right)$ in the OLTG program. Second, the water quality in the MSTG program was better than that in the OLTG program because different rearing models were used (high water exchange model vs. low water exchange model). In addition, the fact that the $c$ effect was not included in Model 2 for the OLTG program may have reduced the direct genetic correlation between the OLGT and MSTG programs. This is because the DGE in the OLTG program may include a component due to the $c$ effect, which does not correlate with the DGE in the MSTG program. 
The accuracy of estimated TBV can be improved using a structure consisting of repeated complete full-sib family groups [25]. However, the DGE and IGE will be completely confounded in such designs, and the associated genetic parameters cannot be estimated for this structure. For the DGE, the optimal design would be one (or a few) large $\operatorname{tank}(\mathrm{s})$ that contain all the families. However, in the 3FAM design, only one family was tested against six other families in three different tanks to estimate IGE [22]. Therefore, the accuracy of DGE would be decreased with MSTG compared to OLTG. Well-designed breeding programs are needed to improve the accuracy of the simultaneous estimations of direct and indirect genetic effects. DGE and IGE were probably more accurate in a design with blocks composed of multiple full-sib families per block. With the block design, each family was combined precisely once with each of the other families in the same block $[6,7]$.

Compared with large livestock and fish breeding programs (e.g., dairy cattle, pig and salmonids) [5, 22, 25], genetic evaluation of IGE may be more feasible in shrimp because shrimp have large full-sib family sizes, a small body size and short production cycle. IGE effects should therefore be considered in real shrimp breeding programs. In the shrimp breeding program used here, shrimp were communally reared in one or several large tanks for testing growth and other traits. Moreover, each family was backed up and reared in a separate tank to avoid the high death risk due to disease and management in the communal rearing population. Therefore, estimating IGE using the MSTG program is straightforward and feasible. Candidates can be selected based on their own performance in the communal population and their sib information from the population of IGE testing.

\section{Conclusions}

In this study, we found a large total heritable variance, which suggested that social interactions may contribute to a large part of the heritable variation in body weight in L. vannamei. Genetic evaluation of IGE may be feasible and should be included in real shrimp breeding programs. The small and non-significant direct-indirect genetic correlation that we estimated implies that neutral or slightly cooperative heritable interactions rather than competition were dominant in this population at a low rearing density. In future experiments, we shall test for IGE at high rearing density and under restricted feeding to quantify direct-indirect genetic covariance.

\section{Authors' contributions}

$\mathrm{SL}$ performed the statistical analysis and wrote the manuscript. $\mathrm{SL}, \mathrm{KL}, \mathrm{ZC}$, and JK conceived and designed the experimental procedure. BXC, XHM, NL, $X \mathrm{~L}$ and SYX participated in producing, managing and testing the families of Litopenaeus vannamei. All authors read and approved the final manuscript.

\section{Author details}

Key Laboratory for Sustainable Utilization of Marine Fisheries Resources, Ministry of Agriculture, Yellow Sea Fisheries Research Institute, Chinese Academy of Fishery Sciences, Nanjing Road 106, Qingdao 266071, China. ${ }^{2}$ Function Laboratory for Marine Fisheries Science and Food Production Processes, Qingdao National Laboratory for Marine Science and Technology, No. 1 Wenhai Road, Aoshanwei Town, Jimo, Qingdao, China. ${ }^{3}$ Hainan Higene Aquaculture Technology Ltd., Wengtian Town, Wenchang 571328, China.

\section{Acknowledgements}

This work was supported by grants from the Special Fund for Basic Scientific Research Business of Central Public Research Institutes (2015B04XK01), the National Natural Science Foundation of China (31572616), the Taishan scholar program for seed industry, the National High Technology Research and Development Program of China (2012AA10A404), the Qingdao Scientific Research Foundation (14-2-4-52-jch5), and the Jiangsu Province Science and Technology Support Project (BE2014414). The authors would also like to thank Dr. Xiaojuan Cao (Huazhong Agriculture University) and Dr. Juan Sui (Yellow Sea Fisheries Research Institute) for their suggestions in the revision of this manuscript.

\section{Competing interests}

The authors declare that they have no competing interests.

Received: 20 May 2015 Accepted: 28 October 2015

Published online: 14 December 2015

\section{References}

1. Muir WM. Incorporation of competitive effects in forest tree or animal breeding programs. Genetics. 2005;170:1247-59.

2. Ellen ED, Muir WM, Teuscher F, Bijma P. Genetic improvement of traits affected by interactions among individuals: sib selection schemes. Genetics. 2007;176:489-99.

3. Zhang P, Zhang X, Li J, Meng Q. Observation of behavior in Fenneropenaeus chinensis and Litopenaeus vannamei postlarvae. J Fish China. 2008;32:223-8.

4. Abdussamad EM, Thampy DM. Cannibalism in the tiger shrimp Penaeus monodon Fabricius in nursery rearing phase. J Aquacult Trop. 1994:9:67-75.

5. Nielsen HM, Monsen BB, Ødegård J, Bijma P, Damsgård B, Toften H, et al. Direct and social genetic parameters for growth and fin damage traits in Atlantic cod (Gadus morhua). Genet Sel Evol. 2014;46:5.

6. Khaw HL, Ponzoni RW, Yip HY, Aziz MA, Bijma P. Genetic and non-genetic indirect effects for harvest weight in the GIFT strain of Nile tilapia (Oreochromis niloticus). Aquaculture. 2016:450:154-61.

7. Bijma P. Estimating indirect genetic effects: precision of estimates and optimum designs. Genetics. 2010;186:1013-28.

8. Muir WM, Bijma P, Schinckel A. Multilevel selection with kin and non-kin groups, experimental results with Japanese quail (Coturnix japonica). Evolution. 2013;67:1598-606.

9. Bijma P, Muir WM, Van Arendonk JA. Multilevel selection 1: quantitative genetics of inheritance and response to selection. Genetics. 2007; 175:277-88.

10. Muir WM. Group selection for adaptation to multiple-hen cages: selection program and direct responses. Poult Sci. 1996;75:447-58.

11. Craig JV, Muir WM. Group selection for adaptation to multiple-hen cages: beak-related mortality, feathering, and body weight responses. Poult Sci. 1996;75:294-302.

12. Griffing B. Selection in reference to biological groups I. Individual and group selection applied to populations of unordered groups. Austr J Biol Sci. 1967;20:127-39.

13. Griffing B. Selection in reference to biological groups. VI. Analysis of full sib groups. Genetics. 1976;82:723-31.

14. FAO. FishStatJ a tool for fishery statistical analysis. Release: 2.12.4. 2015 http://www.fao.org/fishery/statistics/software/fishstatj/en. Accessed 1 June 2015.

15. Gitterle T, Ødegård J, Gjerde B, Rye M, Salte R. Genetic parameters and accuracy of selection for resistance to White Spot Syndrome Virus (WSSV) 
in Penaeus (Litopenaeus) vannamei using different statistical models. Aquaculture. 2006;251:210-8.

16. Gitterle T, Johansen H, Erazo C, Lozano C, Cock J, Salazar M, et al. Response to multi-trait selection for harvest weight, overall survival, and resistance to white spot syndrome virus (WSSV) in Penaeus (Litopenaeus) vannamei. Aquaculture. 2007;272:S262.

17. Cuéllar-Anjel J, White-Noble B, Schofield P, Chamorro R, Lightner DV. Report of significant WSSV-resistance in the Pacific white shrimp, Litopenaeus vannamei, from a Panamanian breeding program. Aquaculture. 2012;368-69:36-9.

18. Sui J, Luan S, Luo K, Meng XH, Lu X, Cao BX, et al. Genetic parameters and response to selection for harvest body weight of Pacific white shrimp, Litopenaeus vannamei. Aquacult Res. 2015. doi:10.1111/are.12729.

19. Huang YC, Yin ZX, Weng SP, He JG, Li SD. Selective breeding and preliminary commercial performance of Penaeus vannamei for resistance to white spot syndrome virus (WSSV). Aquaculture. 2012;364-5:111-7.

20. Luan S, Wang JY, Yang GL, Luo K, Chen XF, Gao Q, et al. Genetic parameters of survival for six generations in the giant freshwater prawn Macrobrachium rosenbergii. Aquacult Res. 2015;46:1345-55.

21. Ponzoni RW, Khaw HL, Nguyen NH, Hamzah A. Inbreeding and effective population size in the Malaysian nucleus of the GIFT strain of Nile tilapia (Oreochromis niloticus). Aquaculture. 2010;302:42-8.
22. Ødegård J, Olesen I. Comparison of testing designs for genetic evaluation of social effects in aquaculture species. Aquaculture. 2011;317:74-8.

23. Gilmour AR, Gogel BJ, Cullis BR, Welham SJ, Thompson R. ASReml user guide. Release 4.1 structural specification. Hemel Hempstead: VSN International Ltd; 2014.

24. Nielsen HM, Ødegård J, Olesen I, Gjerde B, Ardo L, Jeney G, et al. Genetic analysis of common carp (Cyprinus carpio) strains: I: genetic parameters and heterosis for growth traits and survival. Aquaculture. 2010;304:14-21.

25. Ellen ED, Rodenburg TB, Albers GAA, Bolhuis JE, Camerlink I, Duijvesteijn $\mathrm{N}$, et al. The prospects of selection for social genetic effects to improve welfare and productivity in livestock. Front Genet. 2014:5:377.

26. Gitterle T, Rye M, Salte R, Cock J, Johansen H, Lozano C, et al. Genetic (co)variation in harvest body weight and survival in Penaeus (Litopenaeus) vannamei under standard commercial conditions. Aquaculture. 2005;243:83-92

27. Castillo-Juárez H, Casares JCQ, Campos-Montes G, Villela CC, Ortega AM, Montaldo HH. Heritability for body weight at harvest size in the Pacific white shrimp, Penaeus (Litopenaeus) vannamei, from a multi-environment experiment using univariate and multivariate animal models. Aquaculture. 2007;273:42-9.

\section{Submit your next manuscript to BioMed Central and take full advantage of:}

- Convenient online submission

- Thorough peer review

- No space constraints or color figure charges

- Immediate publication on acceptance

- Inclusion in PubMed, CAS, Scopus and Google Scholar

- Research which is freely available for redistribution

Submit your manuscript at

www.biomedcentral.com/submit

(O) Biomed Central 